\title{
Clinical competencies of emergency medical services paramedics in responding to emergency conditions using Objective Structured Clinical Examination (OSCE) in Guilan
}

\section{Arash Amirrafiei, Seyyed Mahdi Zia Ziabari, Fatemeh Haghshenas-Bakerdar, Ehsan Kazemnejad-Leili, Payman Asadi* ${ }^{\mathbb{D}}$}

Guilan Road Trauma Research Center, Guilan University of Medical Sciences, Rasht, Iran

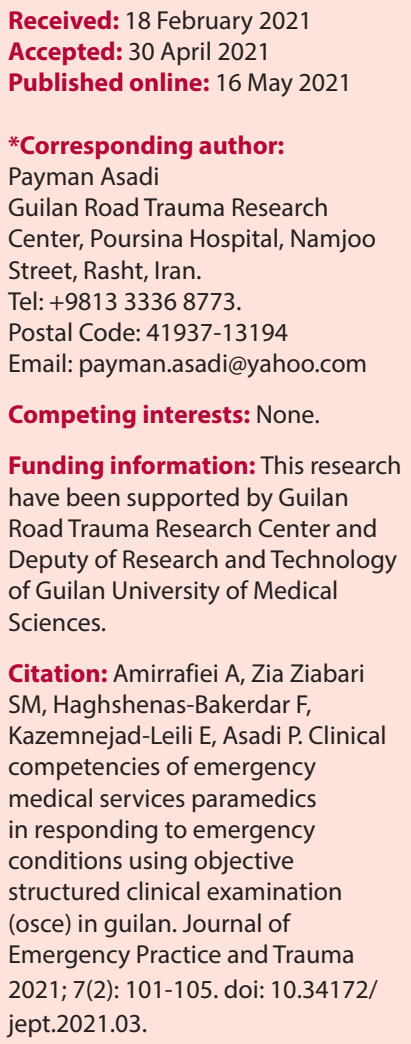

\begin{abstract}
Objective: Evaluating the abilities of emergency medical services (EMS) staff who are in the frontline of the diseases could be an excellent reflection of the accuracy of curriculum both before and after graduation. This study was done to determine the clinical competencies of Guilan EMS staff in responding to emergency conditions using Objective Structured Clinical Examination (OSCE).

Methods: In this descriptive study, 70 EMS staff from selected Emergency Centres in Guilan were recruited. Data were collected using a questionnaire and a checklist which included 9 different skills. Validity of the checklist was assessed by obtaining the opinions of 10 experts. The content validity index (CVI) and content validity ratio (CVR) of the checklist were 0.7 and 0.8 , respectively. The reliability of the checklist was obtained using the test-retest method $(r=0.89)$. In order to collect data, observations were done using the designated checklist. Data were analysed using SPSS software version 22 and descriptive statistical tests.

Results: Findings showed that $56.3 \%$ of the paramedics got good scores for trauma competency but the mean scores for two competencies of spinal cord immobilization and vehicle extrication were low, indicating major skills problem. There was a statistically significant relationship between education $(P=0.02)$ and work experience $(P=0.03)$ as well as clinical skills in confronting trauma.

Conclusion: Although the EMS staff had an acceptable range of performance in most of the skills, it seems that there is a need for training of performance-based competencies in which paramedics had a poor performance.

Keywords: Emergency medical services, Clinical competency, Trauma, Paramedics, Iran
\end{abstract}

\section{Introduction}

In today's modern industrial societies, trauma is one of the most important issues in most countries. According to the World Health Organization (WHO), injuries caused by trauma are the main reasons for death in different countries and up to 2020, trauma will be the third cause of death and disability in different age groups (1). Emergencies are high-risk situations in which individuals' physical or mental conditions are suddenly affected, requiring immediate and appropriate actions to be taken $(2,3)$. To reduce the morbidity and mortality rate of trauma, an appropriate planning for the care and treatment of these patients is needed. Such a planning covers a wide range of treatments, from pre-hospital management to rehabilitation centers. At this stage, an initial diagnosis of the lesions, triage of the injured and early resuscitation occurs. The successful operation of the pre-hospital system will play a crucial role in patients' outcome $(1,3)$. The most critical and important part of the emergency medical services (EMS) is the competent paramedics (4-6). EMS staff perform extensive activities such as airway management, trauma assessment, advanced cardiopulmonary resuscitation, immobilizing patients, dressing, intravenous (IV) therapy, and other advanced 
skills (7). Key skills in delivering pre-hospital care include rapid communication and immediate response, proper evaluation of injured people in different ways, effective treatment, and rapid transfer of the victims to the hospital center (8). According to Dyson et al, most of surveyed EMS staff did not have an appropriate level of pre-hospital competency (9). So, it is necessary to periodically evaluate the competencies of EMS personnel (10). The Objective Structured Clinical Examination (OSCE) is a useful method to assess both physical and knowledge-based skills. In this approach, assessment is conducted at a series of stations with one or two aspects of competence being tested at each station (11). In each station participants must address and resolve various simulated clinical situations that are representative of real clinical practice (12). Since its application in 1975 by Harden et al, OSCE is an effective method and many universities use it to evaluate their students' clinical performance (13). Due to the limited number of studies concerning the competencies of pre-hospital EMS staff, this study was designed to determine the clinical competencies of Guilan EMS staff in responding to emergency conditions using OSCE.

\section{Methods}

This descriptive cross-sectional study was undertaken on 70 EMS staff in the capital of Guilan (Rasht) who were selected by the convenience sampling method. Inclusion criteria included: having a minimum of undergraduate or bachelor degree in medical emergencies and willingness to participate in the study. Exclusion criteria were any psychological disorders in participants.

After obtaining informed consent from participants, in order to survey EMS staff competencies, we designed nine OSCE stations in the skill lab of the school of medicine. So, nine core skills including trauma and triage examination, airway management, carrying the injured victim, vehicle extrication, spinal cord immobilization, venipuncture, life-saving measures (controlling bleeding and shock), injured joint immobilization, and injured bone immobilization were evaluated. Each station time was 10 minutes. To check the performance of the subjects at each station, a performance checklist was designed based on a review of the literature. The validity of the questionnaire was measured by content validity and calculating content validity index (CVI) (0.7) and content validity ratio (CVR) (0.8). A test-retest method was used to assess the reliability of the questionnaire. The questionnaires were administered to 15 EMS staff and were completed twice in 10-day intervals. Then the correlation was calculated (0.89).

To collect data, the main researcher (AA) used the designed checklist at the stations to observe and record the performance of the participants. Each station's score was determined based on the number of items considered for the checklist in each category. In each of the checklists, the score was divided into weak, medium and good levels by dividing the total score of each station by 30 40, 41-80 and 81-122 and they were considered as weak, medium and good scores, respectively. To evaluate the test performance of each station, an evaluator was selected before the test was performed and full details were given on how to complete the checklist. After collecting data, based on the questionnaires, information was entered into SPSS software version 22. Descriptive statistics (standard deviation and mean) and inferential statistical tests (chisquare tests) were used for data analysis.

\section{Results}

The findings of this study showed that the mean age of paramedics was $32 \pm 1.1$ and the mean of their work experience was 7.2 \pm 1.3 . Other demographic information is depicted in Table 1.

The findings on participants' average performance scores on triage skills, airway management, life-saving measures, and some basic clinical skills are summarized in Table 2. According to this table, EMS staff in two skills of spinal cord immobilization and vehicle extrication received low mean scores and had major skills problem.

Furthermore, based on chi-square test, a significant relationship was observed between the performance scores of EMS staff at different stations and their educational level $(P=0.02)$ and work experience $(P=0.03)$ (Table 3$)$.

According to Table 4, 56.3\% of participants had weak clinical competency and $31.3 \%$ had medium clinical competency and none of them had good clinical competency.

\section{Discussion}

This study explored the level of competency of paramedics working in EMS bases in the capital of Guilan to perform nine basic core skills. The findings of the current study indicated that the competencies of paramedics were at low to moderate levels and generally the lowest scores

Table 1. Baseline information of paramedics

\begin{tabular}{llcc}
\hline Variable & & Frequency & Percent \\
\hline \multirow{2}{*}{ Marriage status } & Single & 23 & 32.85 \\
& Married & 47 & 67.14 \\
& Associate degree & 43 & 61.42 \\
Education & Bachelor's degree & 27 & 38.57 \\
& Master's degree & 0 & 0 \\
Field of study & Nursing & 20 & 28.57 \\
& Medical emergency & 41 & 58.57 \\
& Other & 9 & 12.85 \\
& Below 6 months & 9 & 12.85 \\
Work experience & 6-12 months & 18 & 25.71 \\
& 1-5 years & 6 & 8.57 \\
& Above 5 years & 37 & 52.85 \\
\hline
\end{tabular}


Table 2. Average performance scores of the skills examined using the OSCE test

\begin{tabular}{|c|c|c|c|c|}
\hline Evaluated skills & Station total score & $\begin{array}{c}\text { The lowest score } \\
\text { achieved }\end{array}$ & $\begin{array}{c}\text { The highest score } \\
\text { achieved }\end{array}$ & $\begin{array}{c}\text { Mean + SD of the } \\
\text { scores achieved }\end{array}$ \\
\hline Trauma evaluation & 40 & 13 & 39 & $23.2 \pm 2.1$ \\
\hline Airway management & 10 & 5 & 8 & $7.1 \pm 1.1$ \\
\hline Life-saving measures (controlling bleeding and shock) & 12 & 7 & 11 & $9.2 \pm 1.5$ \\
\hline Carrying the injured people & 10 & 3 & 9 & $6.3 \pm 1.1$ \\
\hline Venipuncture & 10 & 2 & 8 & $7.8 \pm 1.2$ \\
\hline Spinal cord immobilization & 10 & 3 & 9 & $5.6 \pm 2.1$ \\
\hline Vehicle extrication & 10 & 2 & 9 & $6.9 \pm 1.7$ \\
\hline Injured joint immobilization & 10 & 4 & 7 & $7.5 \pm 1.6$ \\
\hline Injured bone immobilization & 10 & 5 & 10 & $8.4 \pm 2.5$ \\
\hline
\end{tabular}

Table 3. Relationship between emergency medical staff clinical skills based on demographic variables and work experience

\begin{tabular}{llcccc}
\hline Variable & & $\begin{array}{c}\text { Mean } \pm \text { SD of } \\
\text { clinical skills score }\end{array}$ & $\boldsymbol{F}$ & $\boldsymbol{d f}$ & $\boldsymbol{P}$ value \\
\hline \multirow{2}{*}{ Education } & Associate degree & $92.63 \pm 6.2$ & & & \\
& Bachelor's degree & $100.46 \pm 5.7$ & & 3.21 & 0.02 \\
& Below 6 months & $78.44 \pm 8.2$ & & & \\
Work & $6-12$ months & $77.97 \pm 6.7$ & & & \\
experience & $1-5$ years & $69.44 \pm 7.2$ & & & \\
& Above 5 years & $98.22 \pm 5.4$ & & & \\
\hline
\end{tabular}

Table 4. Distribution of the EMS Staff according to the total score of clinical skills

\begin{tabular}{lccc}
\hline Clinical competency & Number & Percent & Mean \pm SD \\
\hline Weak & 45 & 56.3 & \\
Medium & 25 & 31.3 & \\
Good & 0 & 0 & $99.48 \pm 6.13$ \\
Total & 70 & 100 & \\
\hline
\end{tabular}

were related to spinal column immobilization and vehicle extrication. According to the study conducted by Shakeri et al, it was found that paramedics were poorly trained in two skills namely, immobilization of spinal column and use of traction splint (14). This finding is not consistent with our research and special training should be provided to EMS in this regard and its importance should be emphasized. Based on the study by Aliakbari et al, it was found that the competencies of nurses in performing these skills were at low to moderate levels and special attention should be paid to this area (15). But according to another study by Shakeri et al., the majority of participants (81.1\%) had an average level of trauma knowledge. The present study found a significant statistical relationship between the level of experience $(P=0.02)$ and education level $(P=0.03)$ of participants when confronting trauma. We observed no significant relationship with other demographic characteristics $(\mathrm{P}>0.05)$. In this regard, Aliakbari et al observed no significant correlation between the demographic characteristics of participants and their clinical skills (15). But studies done by Shakeri et al, and Norouzinia et al., showed a significant difference between age, work experience, education level and educational background of paramedics based on their clinical trauma skills $(\mathrm{P}<0.05)$. This finding is consistent with our results $(14,16)$. The study undertaken by Norouzinia et al showed no correlation between work experience and knowledge of trauma. However, our findings indicated a significant and direct relationship between work experience and clinical skills. The reason for this discrepancy may be due to the difference in objectives of that study (16). Shakeri et al observed that education, age, and work experience of EMS staff are effective indicators of skills when confronting trauma (14). According to their findings, there was a significant but inverse correlation between the clinical skills of EMS staff and their age, which is consistent with our results. This can be assumed that as EMS staff become older, their mastery over clinical skills is reduced. One reason for this may be the presence of more experienced and older EMS staff on the outskirts of the city with few missions since the mastery over skills is reduced in the course of time if they are not practiced. Emergency missions are not separated by the age and the background experience of the EMS staff, and paramedics must be able to perform the skills appropriately and wherever and whenever they are. Therefore, it is necessary to pay special attention to further training of this group of employees. Our findings, as well as those of Shakeri et al (14), show that higher education is not a reason for having better clinical skills in the pre-hospital setting, as EMS staff have better mastery over clinical skills than physicians and nurses. This may be due to the education they receive during their studies. High school graduates had the lowest scores for clinical skills among other EMS staff (17). In this regard, Garza et al, who compared the performance of basic-level emergency medical technicians with advanced medical emergency technicians on the ability of decision making on protocols of spinal column immobilization in patients, showed that this skill was similar in both groups. They also found that by providing the necessary training, some skills can be taught to 
basic-level technicians (18). Moreover, Parsa Yekta et al compared the outcome of transferring traumatic patients to the hospital by helicopter in two groups of emergency medical technicians and nurses. They observed that the outcome of patients in both groups was the same. As a result, there was no difference in the performance and skills of nurses and advanced emergency technicians regarding trauma patients (19). In this regard, Garza et al, examined the impact of experience on advanced artificial airway deployment by technicians in the emergency, and found no significant difference between work experience and success of oral-pharyngeal airway deployment by technicians (18). By the same token, Parsa Yekta et al stated that experience is a dynamic concept, not a static one. In other words, if one wants to learn and improve his or her clinical competence, he or she must take an active part in practice. So, paramedics should improve their clinical experience in order to manage different situations (19). It was also found that $56.3 \%$ of participants had a good skill level and $31.3 \%$ had moderate skill levels. None of the participants had a low level of proficiency. In this regard, the study by Shakeri et al. showed that most of the participants (133) had good skills in trauma care and $37.6 \%$ of participants $(80 \%)$ had moderate clinical skills and none of them had a poor level of skill, which is in line with our results. In this regard, the results of a study by Kumar et al, showed that the performance of research units on pre-hospital and emergency care was lower than the average (20).

\section{Limitations of the study}

This study had its own limitations. We evaluated core clinical skills in different OSCE stations which is not a real situation. Therefore, we suggest exploring mastery over clinical skills in real and non-emergency circumstances.

\section{Conclusion}

Paramedics are the first people who arrive at the accident scene and save lives. Based on the obtained results, there is a need for skill training concerning pre-hospital emergency services as paramedics have intermediate proficiency in some of the skills and some of them have poor performance. So, improving the curriculum of emergency medical students is needed.

\section{Ethical Issues}

The EMS staff participating in this study gave their informed consent. The personal data of participants has remained confidential and the results of the study have been reported without divulging the data. This research is part of a doctoral thesis approved by the Vice-Chancellor for Research and Technology of Guilan University of Medical Sciences with No. 38/970322 and code of ethics IR.GUMS.REC.1397.287.

\section{Authors' Contributions}

AA: Study design, data collection, manuscript preparation. SMZZ: Study design, Supervision, data collection, manuscript preparation. FHB: Study design, data collection, manuscript preparation. EKL: Statistical analysis of data, manuscript preparation.

PA: Study design, Supervision, data collection, manuscript preparation.

\section{Acknowledgments}

The researchers would like to offer their special thanks to Dr. Vahid Monsef Kasmaei and all of the study participants and contributors.

\section{References}

1. Norouzinia R, Sharifi A, Bahramian M, Esmaeili Abdar M. The clinical skills of emergency medical staff confronting trauma. Natl J Physiol Pharm Pharmacol 2018; 8(5): 750-4. doi: 10.5455/njppp.2018.8.1249727012018.

2. Alikhanizadeh H, Sabouhi F, Haghani F, Yusefi HA. A study of emergency medical technicians' cognitive and practical skills in airway management and its relationship with some related factors in emergency medical centers selected in Isfahan in the year 2015-2016. Ann Trop Med Public Health 2017; 10(6): 1691-7.doi: 10.4103/atmph.atmph_586_17.

3. Shakeri K, Fallahi-Khoshknab M, Khankeh H, Hosseini M, Heidari M. Knowledge, attitude, and clinical skill of emergency medical technicians from Tehran emergency center in trauma exposure. Int J Crit Illn Inj Sci 2018; 8(4): 188-93. doi: 10.4103/ijciis.ijciis_33_18.

4. Bayrami R, Ebrahimipour H, Rezazadeh A. Challenges in pre-hospital emergency medical service in Mashhad: a qualitative study. Hospital 2017; 16(2): 82-90. [In Persian].

5. Khorasani-Zavareh D, Mohammadi R, Bohm K. Factors influencing pre-hospital care time intervals in Iran: a qualitative study. J Inj Violence Res 2018; 10(2): 83-90. doi: 10.5249/jivr.v10i2.953.

6. Mohammadi M, Firouzkouhi M, Abdollahimohammad A, Shivanpour M. The challenges of pre-hospital emergency personnel in Sistan area: a qualitative study. J Qual Res Health Sci 2020; 8(3): 221-32. [In Persian].

7. Aminizadeh M, Rasouli Ghahfarokhi SM, Pourvakhshoori N, Beyramijam M, Majidi N, Shahabi Rabori MA. Comparing the effects of two different educational methods on clinical skills of emergency intermediate technician: a quasi-experimental research. J Educ Health Promot 2019; 8: 54. doi: 10.4103/jehp.jehp_323_18.

8. Panchal AR, Finnegan G, Way DP, Terndrup T. Assessment of paramedic performance on difficult airway simulation. Prehosp Emerg Care 2020; 24(3): 411-20. doi: 10.3109/10903127.2015.1102993.

9. Dyson K, Bray JE, Smith K, Bernard S, Straney L, Finn J. Paramedic resuscitation competency: a survey of Australian and New Zealand emergency medical services. Emerg Med Australas 2017; 29(2): 217-22. doi: 10.1111/17426723.12715 .

10. Horrocks P, Hobbs L, Tippett V, Aitken P. Paramedic disaster health management competencies: a scoping review. Prehosp Disaster Med 2019; 34(3): 322-9. doi: 10.1017/s1049023x19004357. 
11. Duanmu Y, Henwood PC, Takhar SS, Chan W, Rempell JS, Liteplo AS, et al. Correlation of OSCE performance and point-of-care ultrasound scan numbers among a cohort of emergency medicine residents. Ultrasound J 2019; 11(1): 3. doi: 10.1186/s13089-019-0118-7.

12. Harden RM, Lilley P, Patricio M. The Definitive Guide to the OSCE: The Objective Structured Clinical Examination as a Performance Assessment. 4th ed. New York: Elsevier; 2016.

13. Solà-Pola M, Morin-Fraile V, Fabrellas-Padrés N, RaurellTorreda M, Guanter-Peris L, Guix-Comellas E, et al. The usefulness and acceptance of the OSCE in nursing schools. Nurse Educ Pract 2020; 43: 102736. doi: 10.1016/j. nepr.2020.102736.

14. Shakeri K, Fallahi Khoshknab M, Khankeh H, Hosseini M, Hosseinzadeh S, Haghi Monie N. Evaluation of clinical skills of medical emergency personnel in Tehran Emergency Center confronting the trauma. Journal of Health Promotion Management 2012; 1(4): 16-24. [In Persian].

15. Aliakbari F, Aien F, Bahrami M. Assessment competencies among emergency nurses for responding in disaster situation with Objective Structured Clinical Examination.
Journal of Health Promotion Management 2014; 3(3): 4757. [In Persian].

16. Norouzinia R, Ahmadi M, Seidabadi M. Knowledge and clinical competence of medical emergencies students in facing trauma. Iranian Journal of Emergency Medicine 2016; 3(2): 77-3. doi: 10.22037/ijem.v3i2.10641.

17. Kim JH, Choi ES. Core competency in disaster management of 119 paramedics. The Korean Journal of Emergency Medical Services 2017; 21(3): 35-48.

18. Garza AG, Gratton MC, Coontz D, Noble E, Ma OJ. Effect of paramedic experience on orotracheal intubation success rates. J Emerg Med 2003; 25(3): 251-6. doi: 10.1016/s07364679(03)00198-7.

19. Parsa Yekta Z, Ahmadi F, Tabari R. Factors defined by nurses as influential upon the development of clinical competence. Journal of Guilan University of Medical Sciences 2005; 14(54): 9-23. [In Persian].

20. Kumar S, Agarwal AK, Kumar A, Agrawal GG, Chaudhary S, Dwivedi V. A study of knowledge, attitude and practice of hospital consultants, resident doctors and private practitioners with regard to pre-hospital and emergency care in Lucknow. Indian J Surg 2008; 70(1): 14-8. doi: 10.1007/s12262-008-0003-2. 